\title{
Study of coherent pion production in proton-deuteron collisions with polarized beams and target at ANKE-COSY
}

\author{
S. Dymov ${ }^{1,2, \star}$ and V. Shmakova ${ }^{2}$ \\ for the ANKE Collaboration \\ ${ }^{1}$ Institut für Kernphysik, Forschungszentrum Jülich, D-52425 Jülich, Germany \\ ${ }^{2}$ Laboratory of Nuclear Problems, Joint Institute for Nuclear Research, 141980 Dubna, Russia
}

\begin{abstract}
Values of the proton analysing power, deuteron vector analysing power, and the deuteron-proton spin correlations in the $p d \rightarrow{ }^{3} \mathrm{He} \pi \pi^{0} /{ }^{3} \mathrm{H} \pi^{+}$reactions at $350-360 \mathrm{MeV}$ per nucleon were obtained by using polarized beams and target at ANKE-COSY. Data were also obtained on the deuteron-proton spin correlation and proton analysing power at small angles at $600 \mathrm{MeV}$ per nucleon. By combining the extrapolated values of the spin correlations to the forward or backward directions with published measurements of the deuteron tensor analysing powers, the relative phases between the two non-vanishing amplitudes were evaluated.
\end{abstract}

\section{Introduction}

Two-body pion production in the interaction of protons with few-nucleon systems is of interest, both from the point of view of studying the reaction mechanism, and from that of determining the structure of light nuclei. The success of microscopic models with explicit $\Delta$-excitation for two-nucleon systems suggests that these models should be tested in the three-nucleon case, where production of $\Delta$ is intimately linked to $3 \mathrm{~N}$ forces. The phenomenological approach, using impulse approximation with $p p \rightarrow d \pi^{+}$cross section, as input was successful near the reaction threshold but only partial progress has been achieved at higher energies [1].

In general six invariant amplitudes are required to describe the $p d \rightarrow{ }^{3} \mathrm{He} \pi^{0}$ reaction and these amplitudes will be functions of the angle between the incident proton and outgoing pion in the c.m. frame. The number of independent functions reduce to two at threshold or in the forward/backward directions. These may be written as [2]

$$
F\left(d p \rightarrow{ }^{3} \mathrm{He} \pi^{0}\right)=\sqrt{\frac{1}{2}} \bar{u}_{\tau} \vec{p} \cdot(A \vec{\epsilon}+i B \vec{\epsilon} \times \vec{\sigma}) u_{p}
$$

Here $\vec{\epsilon}$ is the deuteron polarisation vector, $\vec{p}$ and $\vec{k}$ the proton and pion centre-of-mass momenta and $u_{p}$ and $u_{\tau}$ the initial and final fermion spinors. The amplitude should be multiplied by a $\sqrt{2}$ factor if the $p d \rightarrow{ }^{3} \mathrm{H} \pi^{+}$reaction is being considered.

^e-mail: s.dymov@fz-juelich.de 
If only the two amplitudes $A$ and $B$ are retained, the unpolarised c.m. differential cross section, deuteron tensor analysing power, and vector transverse spin correlation become

$$
\begin{aligned}
\frac{\mathrm{d} \sigma}{\mathrm{d} \Omega} & =\frac{k p}{3}\left(|A|^{2}+2|B|^{2}\right), \\
T_{20} & =\sqrt{2} \frac{|B|^{2}-|A|^{2}}{|A|^{2}+2|B|^{2}}, \\
C_{y, y} & =-\frac{2 \operatorname{Re}\left(A^{*} B\right)}{|A|^{2}+2|B|^{2}},
\end{aligned}
$$

whereas $i T_{11}$ and $T_{22}$, as well as the proton analysing power $A_{y}$, must all vanish.

\section{Spin correlation coefficients}

The $p d \rightarrow{ }^{3} \mathrm{He} \pi^{0}$ and $p d \rightarrow{ }^{3} \mathrm{H} \pi^{+}$reactions have been studied experimentally over many decades and a wealth of data on the differential cross sections and analysing powers has been collected for these processes. However, the double polarisation observables have been explored far less and information on the spin correlations is still very scarce. The ANKE spectrometer [3] equipped with an internal polarised target together with the polarised deuteron beam of COSY synchrotron (Jülich, Germany) offer a unique opportunity to conduct measurements of the transverse spin correlation coefficients in these reactions.

Two double-polarisation experiments have been performed at ANKE with a polarised deuteron beam and a polarised hydrogen target, at the beam energies of 363 and $600 \& 1115 \mathrm{MeV}$ per nucleon. The data at the two lower energies were analysed to obtain the spin correlations in the $p d \rightarrow{ }^{3} \mathrm{He} \pi^{0}$ reaction, and the results have recently been published [4].

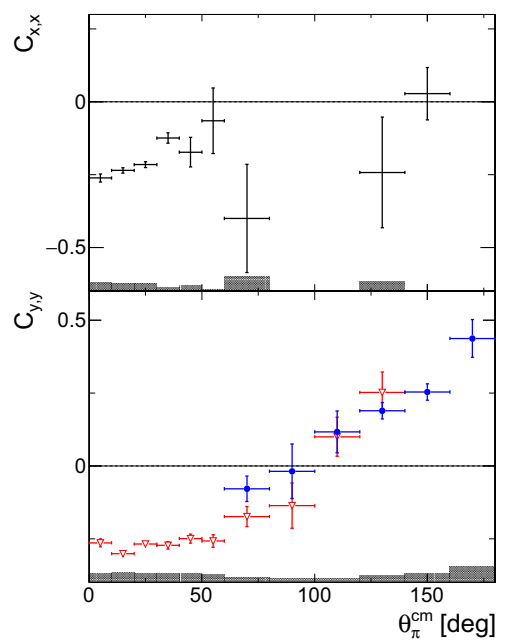

Figure 1. Transverse spin correlation coefficients $C_{x, x}$ and $C_{y, y}$ in the $\vec{d} \vec{p} \rightarrow{ }^{3} \mathrm{He} \pi^{0}$ and $\vec{d} \vec{p} \rightarrow{ }^{3} \mathrm{H} \pi^{+}$reactions at $363 \mathrm{MeV}$ per nucleon [4]. In the $C_{y, y}$ case the (red) inverted triangles were obtained through ${ }^{3} \mathrm{He}$ detection and the (blue) circles through ${ }^{3} \mathrm{H}$ detection. The shaded area indicates the systematic uncertainties in the measurement.

The spin correlations at $363 \mathrm{MeV} / \mathrm{n}$ are presented in Fig. 1. Both ${ }^{3} \mathrm{He}$ and ${ }^{3} \mathrm{H}$ production processes could be investigated at this energy and the results in the angular range of $60-140^{\circ}$ covered by the both reactions are completely compatible. The ANKE detector acceptance is limited by the size of the gap in the analysing magnet D2 and is concentrated around $\phi=0^{\circ}$ and $180^{\circ}$ regions. This leads to a better definition of the $C_{y, y}$ coefficient as compared to $C_{x, x}$. 


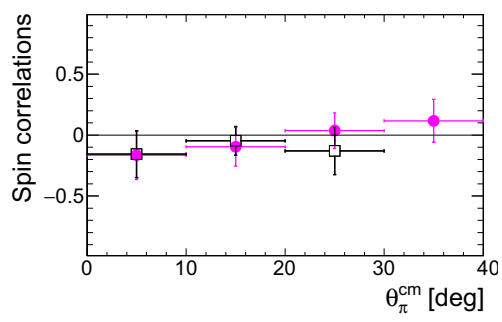

Figure 2. Transverse spin correlation coefficients $C_{y, y}$ (magenta circles) and $C_{x, x}$ (open squares) measured in the $\vec{d} \vec{p} \rightarrow{ }^{3} \mathrm{He} \pi^{0}$ reaction at $600 \mathrm{MeV}$ per nucleon [4]. The systematic errors are below 0.03 .

Since a clean selection of tritium by the energy loss in the scintillation hodoscope is no longer feasible at $600 \mathrm{MeV}$ per nucleon, only data on the $p d \rightarrow{ }^{3} \mathrm{He} \pi^{0}$ reaction are shown at this higher energy. The $C_{x, x}$ and $C_{y, y}$ coefficients measured at small angles are presented in Fig. 2.

The deuteron tensor analysing power $T_{20}$ was measured in the forward/backward directions at Saclay [5] and the values interpolated at $726 \mathrm{MeV}$ are $T_{20}\left(0^{\circ}\right)=-1.01 \pm 0.01$ and $T_{20}\left(180^{\circ}\right)=$ $-1.10 \pm 0.06$. However, it is easily shown from Eq. (2) that the corresponding spin correlation in the forward or backward direction is bounded by

$$
\left(C_{y, y}\right)^{2} \leq \frac{4}{9}\left[1-T_{20} / \sqrt{2}-\left(T_{20}\right)^{2}\right],
$$

from which one sees quite generally that $\left|C_{y, y}\right| \leq 1 / \sqrt{2}$.

Using the forward value of $T_{20}$ measured at Saclay, Eq. (3) shows that $\left|C_{y, y}\left(0^{\circ}\right)\right| \leq 0.56 \pm 0.01$ to be compared to the value $-0.27 \pm 0.03$ deduced from extrapolating the combined data of Fig. 1 to the forward direction. The error bars are larger in the backward direction where one finds from the data of Fig. 1 that $C_{y, y}\left(180^{\circ}\right)=+0.46 \pm 0.04$ compared to the upper bound from Eq. (3) of $0.50 \pm 0.04$.

The change in sign of $C_{y, y}$ between the backward and forward directions is significant. If the phase angle is defined by $\phi=\arg (B / A)$ then, in the forward direction, $\cos \phi=0.49 \pm 0.05$ whereas in the backward direction $\cos \phi=-0.90 \pm 0.10$. The change in sign of $\operatorname{Re}\left(A^{*} B\right)$ could be due to structure in either amplitude. The Saclay data indicate that this is likely to be caused by the $B$ amplitude because $B\left(180^{\circ}\right)$ seems to have a zero in the vicinity of $T_{d}=650 \mathrm{MeV}$ [5].

The deuteron tensor analysing power bound of Eq. (3) provides little real constraint at $1.2 \mathrm{GeV}$. Using the Saclay value of $T_{20}\left(0^{\circ}\right)=-0.66 \pm 0.02$ one finds that $\left|C_{y, y}\left(0^{\circ}\right)\right|<0.68 \pm 0.01$ to be compared with the extrapolated value of Fig. $2, C_{y, y}\left(0^{\circ}\right)=-0.10 \pm 0.08$. These mean that in the forward direction the $A$ and $B$ amplitudes at $600 \mathrm{MeV}$ per nucleon are almost completely out of phase, with $\cos \phi=0.14 \pm 0.12$.

\section{Vector analysing powers}

In addition to the spin correlation parameters, the proton and deuteron vector analysing powers can also be extracted from these data [4]. At $363 \mathrm{MeV} / \mathrm{n}, A_{y}^{p}$ can be compared to the data of Ref. [6] and to the high statistics ANKE measurement with a polarised proton beam and a deuterium cluster-jet target (see Fig. 3). Despite the smooth behaviour of the differential cross sections with angle, the rich structure in $A_{y}^{p}$ indicates that many partial waves with different phases contribute actively at this energy.

Since the tensor polarisation of the deuteron beam was vanishingly small, the deuteron vector analysing power $A_{y}^{d}$ could also be extracted from the data by looking at the dependence of the counting rates on the vector polarisation of the beam. The results obtained from the combined $\vec{d} p \rightarrow{ }^{3} \mathrm{He} \pi^{0} /{ }^{3} \mathrm{H} \pi^{+}$data are shown in Fig. 4. It is perhaps significant that the abrupt change in $A_{y}^{d}$ occurs at $\theta_{\pi}^{c m} \approx 80^{\circ}$, which is close to the deep minimum in $A_{y}^{p}$. 
The available statistics in the $600 \mathrm{MeV} / \mathrm{n}$ case is very limited. The uncertainties in the corresponding deuteron vector analysing powers are very large and these data are not shown. However, values of the proton analysing powers could be extracted for small angles by exploiting the polarisation of the hydrogen in the target and these data are presented in Fig. 5. These results for $\theta_{\pi}^{c m}<40^{\circ}$ are significantly larger than those of the lower energy data shown in Fig. 3.
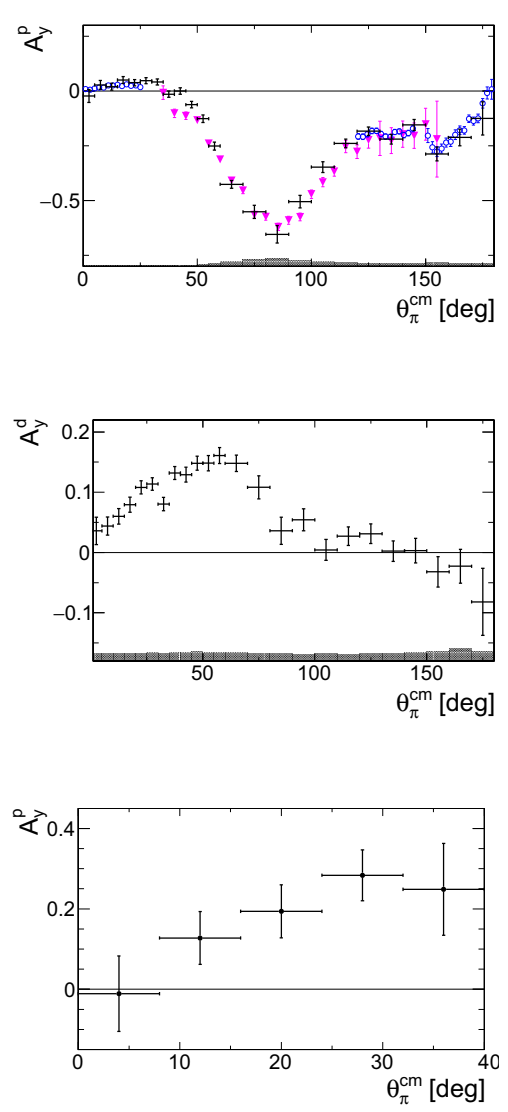

Figure 3. TRIUMF data on the proton analysing power $A_{y}^{p}$ in the $\vec{p} d \rightarrow{ }^{3} \mathrm{He} \pi^{0}$ reaction at $350 \mathrm{MeV}[6]$ (magenta triangles) are compared to ANKE results at $353 \mathrm{MeV}$ [4] (blue open circles). We do not include here the $9 \%$ systematic error that is mainly associated with uncertainties in the COSY beam polarisation. Also shown are the ANKE values at $363 \mathrm{MeV}$ per nucleon [4] deduced from measurements with a deuteron beam incident on a long cell filled with polarised hydrogen gas (black crosses). The shaded area indicates the systematic uncertainties in the deuteron beam measurements.

Figure 4. Deuteron vector analysing power $A_{y}^{d}$ measured in the $\vec{d} p \rightarrow{ }^{3} \mathrm{He} \pi^{0} /{ }^{3} \mathrm{H} \pi^{+}$reactions at $726 \mathrm{MeV}$ [4] The data are presented in terms of the c.m. angle between the proton and pion. The shaded area indicates the systematic uncertainties in the measurement.

Figure 5. The proton analysing power $A_{y}^{p}$ for the $d \vec{p} \rightarrow{ }^{3} \mathrm{He} \pi^{0}$ reaction at $600 \mathrm{MeV}$ per nucleon [4] extracted from data obtained with a polarised hydrogen target. Systematic uncertainties, which were dominated by those in the target polarisation, were below $5 \%$.

We are grateful to the COSY crew for providing such good working conditions, especially of the polarised beam. This work has been partially supported by the the COSY FFE programme and the Shota Rustaveli National Science Foundation.

\section{References}

[1] W. R. Falk, Phys. Rev. C 50, 1574 (1994)

[2] J.-F. Germond, C. Wilkin, J. Phys. G 16, 381 (1990)

[3] S. Barsov et al., Nucl. Instrum. Methods A 462, 364 (1997)

[4] S. Dymov et al., Phys. Lett. B 762, 102 (2016)

[5] C. Kerboul et al., Phys. Lett. B 181, 28 (1986)

[6] J. M. Cameron et al., Nucl. Phys. A 472, 718 (1987) 